\title{
THE RELATION BETWEEN TEXTURE AND MICROSTRUCTURE IN ROLLED FCC MATERIALS
}

\author{
T. Leffers and D. Juul Jensen \\ Materials Department, Risø National Laboratory, DK-4000 Roskilde, Denmark
}

\begin{abstract}
The relations between texture and microstructure in rolled fcc metals and alloys are described. In materials with low stacking fault energy texture and microstructure together provide a consistent empirical description of the deformation process. In materials with intermediate and high stacking fault energy things are more complicated. There are a number of textural and microstructural features which are not understood and which probably require a combination of texture and microstructure to be understood. A substantial effort is needed in order to establish this connection.
\end{abstract}

\section{INTRODUCTION}

It is obvious that there must be a relation between texture and microstructure in deformed materials since they both reflect the deformation pattern. However, for many years the simple (full-constraint) Taylor $\operatorname{model}^{1}$ dominated the discussions of plastic deformation and deformation texture, and this model presupposes rather trivial microstructures. Fig. 1 illustrates this traditional view of the microstructures for fcc materials. In materials with high to intermediate stacking fault energy the only generally accepted microstructural feature was the formation of cells or subgrains which is below the resolution limit of the Taylor model. For materials with low stacking fault energy the Taylor model was supplemented with the formation of bulky deformation twins.

Towards the end of the seventies it became generally accepted that the Taylor constraints are relaxed to some extent depending on conditions, e.g. ref. ${ }^{2}$. Correspondingly, it became generally accepted that deformation heterogeneities at a scale coarser than that of the subgrains were important components of the microstructure. Today there is a large number of observations of such heterogeneities. Thus, the real situation is radically different from that sketched in fig. 1: a number of microstructural features, including the "organized structures" as described in ref. ${ }^{3}$, are basically within the resolution limit of the relevant models for plastic deformation and texture formation. This means that the relation between texture and microstructure is not trivial. Texture and microstructure reveal different aspects of the deformation pattern which we must combine and reconcile in order to reach a proper understanding of the deformation process.

In the present work we discuss the texture/microstructure relations for rolled fcc 
Copper:

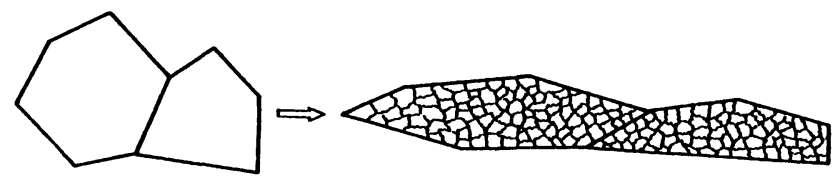

Brass:

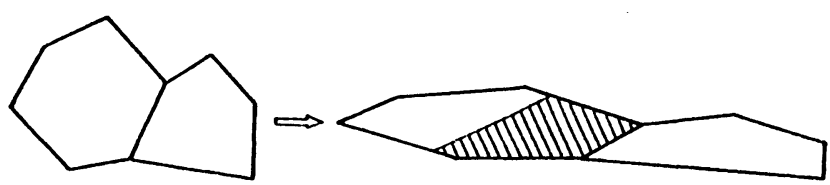

Fig. 1. Trivial microstructures in deformed materials with high- to-intermediate and low stacking fault energy (copper and brass, respectively) as envisaged earlier.

materials. We do not imply that this is representative for all types of materials subjected to all types of strain patterns, but we assume that we point out some important trends. Within the framework of our present knowledge rolled fcc materials represent two extreme cases: for materials with low stacking fault energy texture and microstructure in combination provide a convincing empirical description of the deformation pattern; for materials with higher stacking fault energy, on the other hand, it is not clear how we should combine texture and microstructure. As far as the overall deformation pattern is concerned, the two types of material also represent two extreme cases when subjected to rolling deformation: in materials with low stacking fault energy the Taylor model does not apply, whereas it applies reasonably well (with full or relaxed constraints) in materials with higher stacking fault energy - at least in a statistical sense.

\section{TEXTURES IN MATERIALS WITH LOW STACKING FAULT ENERGY}

It has been stated frequently that the rolling textures in all fcc materials are identical up to about $50 \%$ reduction and that they, in this range of strains, are of the type characteristic of materials with higher stacking fault energy, the copper type. For instance, recent observation by Hirsch et al. ${ }^{4-6}$ are taken to support this point of view. However, a number of observations (on brass, ${ }^{70}$ copper-germanium, ${ }^{11}$ austenitic steel $^{12}$ ) demonstrate that the texture development in materials with low stacking fault energy is different from that in materials with higher stacking fault energy from an earlier stage. Fig. 2 shows the development in orientation density for orientations within $15^{\circ}$ from $\{211\}<111>$ for three different copper materials and three different brass materials (with $15 \%$ zinc). The measurements were made with neutron diffraction with two slightly different experimental set-ups. Some of the results were reported in ref. ${ }^{10}$ One sees a difference in texture development between copper and brass from the very beginning. Also the pole figures and the ODFs clearly reveal this difference - a difference which represents a development towards the respective final copper and brass textures. ${ }^{10}$

The texture in materials with low stacking fault energy is not a Taylor-type texture. Formally the brass-type texture may be obtained with a Taylor deformation 


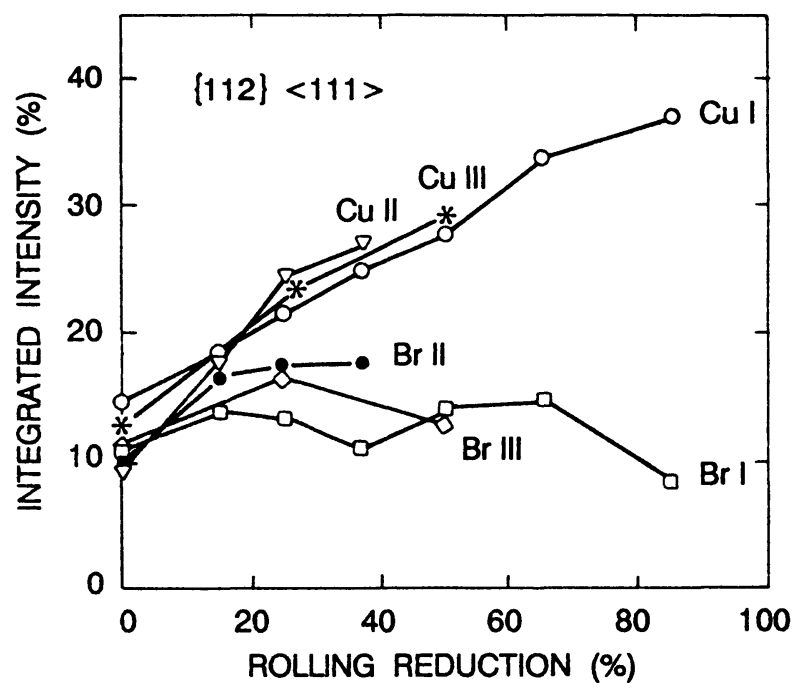

Fig. 2. Evolution with rolling strain of the fraction of material within $15^{\circ}$ from $\{211\}<111>$ for copper and brass (15\% zinc) from 6 different batches.

pattern combined with bulk twin formation (as sketched in fig. 1), e.g. refs. ${ }^{13,14}$, but the actual volume fraction of twinned material is too small to have any significant direct effect on the texture (see next section).

The alternatives to the Taylor model are models with softer interaction between the grains (or between the individual grains and a continuum matrix) as for instance described in ref. ${ }^{3}$; the exact physical reason for the use of models with soft (plastic) interaction between the grains will be given in the next section. Apart from the simple Sachs model, ${ }^{15}$ which is unacceptable because it does not fulfil the continuum requirements (neither in strain or stress), the simplest soft-interaction model is the modified Sachs model (e.g. Pedersen and Leffers ${ }^{16}$ ). In this model the deterministically selected slip systems of the Sachs model are supplemented with slip systems selected with a stochastic procedure to produce multiple slip. The stochastic procedure (not to be described in detail here) is based on rational considerations, i.e. it is not a mere curve-fitting procedure.

Fig. 3 shows $\{111\}$ and $\{200\}$ pole figures for $50 \%$ reduction simulated with the modified Sachs model together with experimental brass pole figures. There is a rather good agreement between the simulated and the experimental pole figures, qualitatively and quantitatively. The only obvious disagreement is that the fibre component with $<100>$ parallel to the rolling direction is too strong in the simulated texture.

We have deliberately chosen to compare simulation and experiment for a moderate degree of reduction like $50 \%$ with a reasonably simple microstructure (as described in the next section). At higher strains the microstructure, and hence the deformation pattern, becomes quite complicated (particularly because of extensive shear banding). This should, theoretically, make texture simulation difficult. In practice the modified Sachs model simulates the final brass-type texture quite well, e.g. ref. ${ }^{17}$; the simulated 
$\mathrm{RD}$

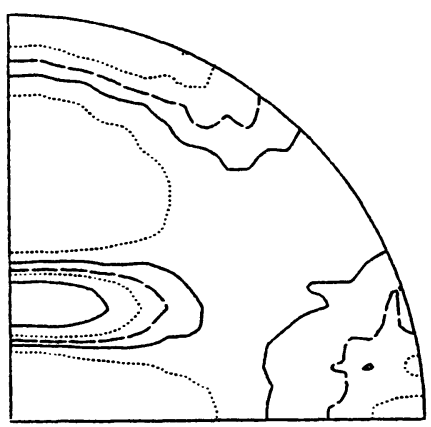

111

$3.0 \quad 2.0 \quad 1.5 \quad 1.0 \quad \ldots .5$

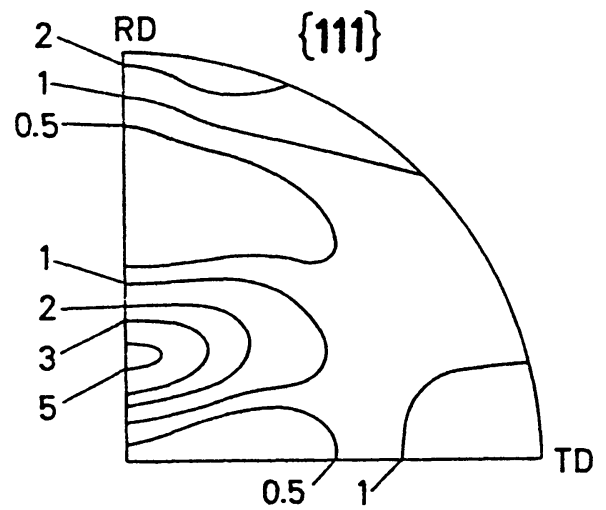

$\mathrm{RD}$

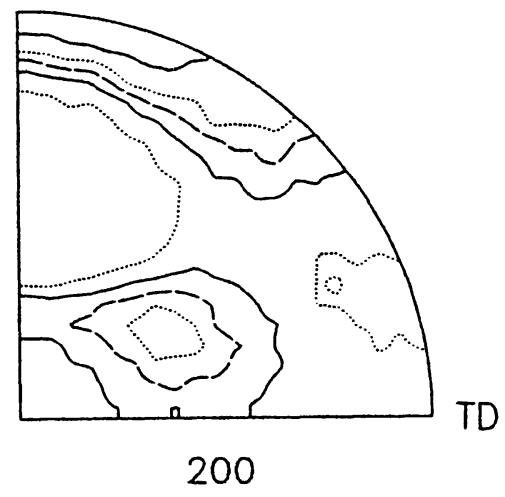

$3.0 \quad 2.0 \quad 1.5 \quad 1.0 \quad 0.5$

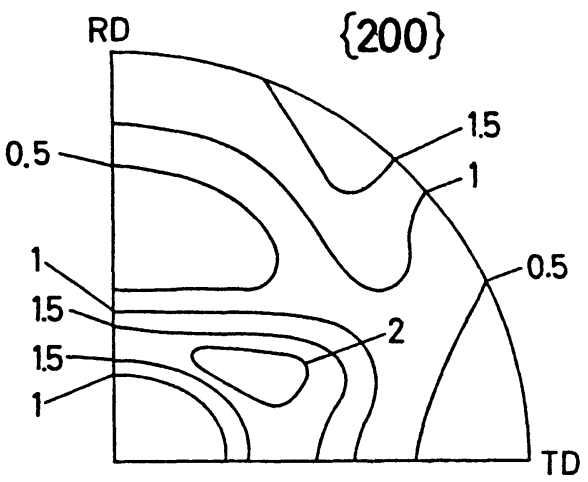

Fig. 3. $\{111\}$ and $\{200\}$ pole figures for $50 \%$ rolling reduction. Top: simulated with the modified Sachs model; bottom: measured for brass with $15 \%$ zinc.

texture reproduces the main trends, viz. the maximum in orientation density at $\{110\}<112>$ with spread to $\{110\}<001>$, but it seriously overestimates the sharpness of the texture. The simulated texture does not reproduce the texture component with $\{111\}$ parallel to the rolling plane - which becomes evident at about $60 \%$ reduction, peaks at about $80 \%$ reduction and remains present up to very high strains.

\section{MICROSTRUCTURES IN MATERIALS WITH LOW STACKING FAULT ENERGY}

Wassermann ${ }^{18}$ introduced the idea that mechanical twins were the important microstructural features in fcc materials with low stacking fault energy as far as texture formation is concerned. Wassermann's concept of twins was as sketched in fig. 1: bulky features which exerted their effect on texture via the volume of differently oriented material that they represent. It is now established beyond any reasonable doubt that this twin concept is not correct. Mechanical twins in fec materials form as very thin lamellae. 
At moderate degrees of deformation ( $\sim 40 \%$ reduction) they only make up a volume fraction of the order of few percents in brass with $15 \%$ zinc. ${ }^{8,19}$ At higher strains in brass with $30 \%$ zinc Duggan, Hatherly, Hutchinson and Wakefield ${ }^{20}$ quote a substantially higher volume fraction, but still a fraction which is insufficient to have a decisive direct effect on texture.

It is correct, on the other hand, that the twin lamellae are predominant microstructural features. At moderate strains, where shear bands do not form, the twin lamellae are actually, apart from the homogeneously distributed dislocations, the only features observed in the microstructure. When the twin lamellae form at low to intermediate strains, they are not distributed homogeneously. As observed for instance by Leffers and Kayworth ${ }^{8}$ and Duggan et al. ${ }^{20}$ and described in detail by Leffers and Bilde-Sørensen, ${ }^{19}$ the twin lamellae cluster to form "bundles" consisting of a composite of twin lamellae and untwinned matrix. An example is shown in fig. 4.

In brass with $15 \%$ zinc rolled to reductions of $\sim 40 \%$, a $0.40-0.45$ fraction of the grains have a structure dominated by bundles. Another $0.40-0.45$ fraction have no or practically no twins. The remaining fraction of the grains contain scattered twins that do not form proper bundles. ${ }^{21}$ Which of the three groups a given grain belongs to, is determined by its orientation. For instance, grains with $\{110\}$ approximately parallel to the rolling plane do not contain bundles. ${ }^{19,21}$ The formation of bundles seems to be favoured by a high maximum resolved shear stress for twinning relative to that for slip and by a high resolved shear stress (for slip) on the most heavily loaded slip system relative to that on the next most heavily loaded system. ${ }^{21}$

Fig. 4 illustrates the normal situation with only one system of bundles in a grain. Occasionally grains with two systems of crossing bundles are observed. ${ }^{19}$ In all these cases one of the systems (system No. 2) produces shear displacement of the other (system No. 1) whereas system No. 1 never produces displacement of system No. 2. These observations, together with observations of grain boundaries shear-displaced by

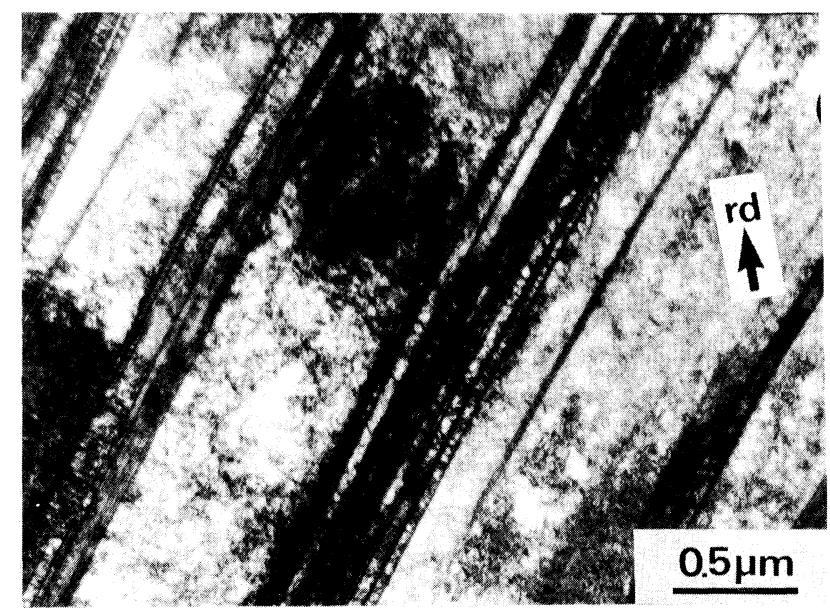

Fig. 4. One system of bundles in brass ( $15 \%$ zinc) rolled to $37 \%$ reduction. Longitudinal section, rolling direction indicated. 
bundles, show that the bundles have a specific micromechanical function: they are the carriers of shear. ${ }^{19}$ The observations on crossing bundles also show that even in grains with two systems of bundles only one system operates as carrier at a given time (a given strain): system No. 1 ceases to operate when system No. 2 is activated. The microscopic deformation pattern which emerges for the grains with bundles at moderate reductions is as follows: ${ }^{19}$

(i) at a given strain shear is concentrated in one system of bundles (and normally there is only one system of bundles throughout the whole range of strains)

(ii) the shear is on the $\{111\}$ plane parallel to the bundle, and it is mainly normal slip since the twin lamellae only make up a minor fraction of the bundles

(iii) as a consequence of (i) there is very little slip in the areas between the bundles - on the slip plane parallel to the bundles and on the slip planes intersecting the bundles.

The point in (iii) about the extent of slip, in the areas between the bundles, on $\{111\}$ planes others than that parallel to (or approximately parallel to) the bundles has been subjected to a detailed investigation. ${ }^{19}$ If there is significant slip on the other $\{111\}$ planes, the twin lamellae in the bundles will deviate from the $\{111\}$ plane on which they formed originally; if there is not, the twin lamellae will remain parallel to their parent $\{111\}$ plane, i.e. zero is the theoretical lower bound for the deviation. A reasonable upper bound for the deviation is that corresponding to a Taylor pattern of multiple slip. Fig. 5 shows the upper-bound deviation distribution as emerging from a Taylor simulation together with the experimental deviation distribution for brass with $15 \%$ zinc. The variation in the theoretical deviation comes from the differently oriented grains in the computer model. The variation in the experimental deviation is the observed grain to grain variation. For details about the computational and the experimental procedures the reader is referred to ref. ${ }^{19}$, but we shall quote one particularly relevant point about the experiments: because of various experimental inaccuracies which we have not corrected for, the real experimental deviation distribution is significantly narrower (closer to zero) than the as observed. It is clear from fig. 5 that the experimental deviation distribution is much closer to the theoretical lower bound (deviation zero for no slip on slip planes intersecting the plane of the twins/bundles) than to the Taylor upper bound - and it is even clearer if one accounts for the experimental inaccuracies.

It should be mentioned that the experimental deviation distribution in fig. 5 is narrower than that quoted in ref. ${ }^{19}$. The reason for the difference is that the present distribution only includes grains with bundles whereas that in ref. ${ }^{19}$ also included grains with scattered twins. However, despite of the somewhat wider distribution in ref. ${ }^{19}$, the conclusion was the same as that in the present work. In many grains the bundles (and the twin lamellae in the bundles) are bent. ${ }^{19}$ In accordance with fig. 5 this does not mean that the bundles bend away from $\{111\}$. It means that the twins and the matrix material bend together.

The overall conclusion for the range of moderate reductions is that the grains with bundles predominantly deform by slip on one single slip plane, viz. that parallel to the bundles. So far, the microstructural observations have not provided much direct information about the slip pattern in the other grains. However, a comparison of fig. 5 with the corresponding figure in ref. ${ }^{19}$ indicates that there is more multiple slip in grains with scattered twins than in grains with bundles. 


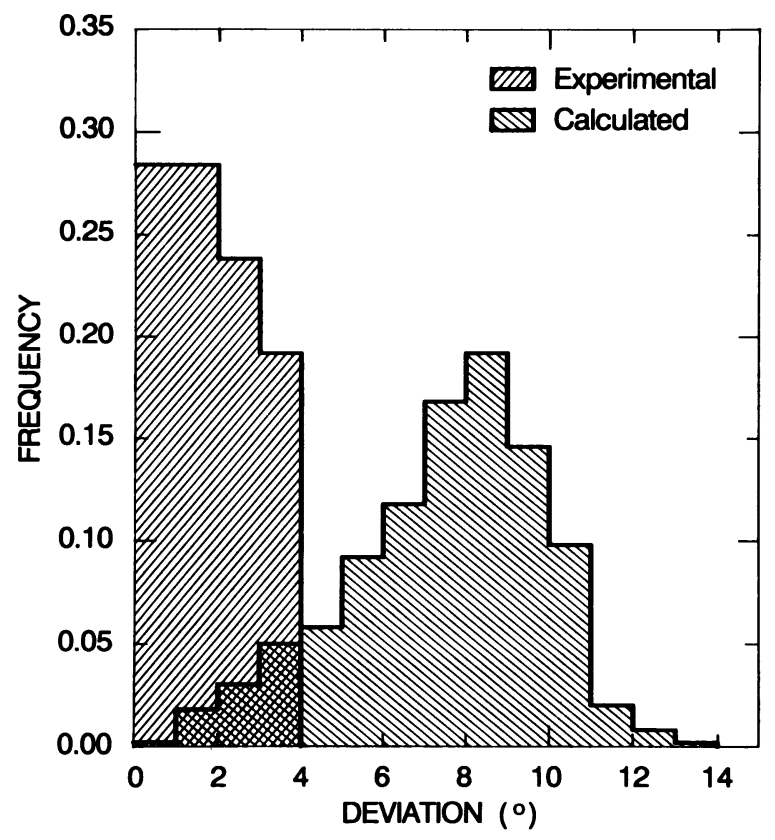

Fig. 5. Distributions of the angular deviation of twin lamellae from the nearest $\{111\}$ plane. The calculated deviation distribution is the upperbound solution derived by Taylor simulation. The experimental distribution represents TEM measurements on brass with $15 \%$ zinc. Both distributions correspond to $37 \%$ rolling reduction.

The overall conclusion above is based on observations on brass with $15 \%$ zinc. However, the scattered microstructural information available for moderate reductions of brass with higher zinc content (e.g. ref. ${ }^{20}$ ) and austenitic steels (e.g. refs. ${ }^{12,22}$ ) indicates that the conclusion is also basically correct for these materials (see ref. ${ }^{19}$ ).

At higher strains the microstructure changes. At $65 \%$ reduction practically all grains in brass with $15 \%$ zinc have a high density of twin lamellae (but still a relatively low volume fraction of twinned material). ${ }^{23}$ The same development seems to take place in other materials with low stacking fault energy. For the grains which contained bundles already at lower strains one must imagine that new bundles or widening preexisting bundles are filling up the grains. For the other grains it is uncertain whether they are filled up with individual (scattered) twin lamellae or with bundles. Grains with twin lamellae (and \{111\}) approximately parallel to the rolling plane become increasingly common, e.g. refs. ${ }^{12,20,22,24}$ There seems to be some contradiction between microstructural and textural observations about exactly how common these grains are: the microstructural observations quoted indicate that they make up the majority of the grains, while the texture component with $\{111\}$ parallel to the rolling plane is not the major texture component. At these strains shear bands form in large numbers. ${ }^{12,20,22,24,25}$ The shear bands erode the twin-matrix composite structure and transforms it to a structure with fine crystallites or subgrains with large orientation spread. 
There is no direct investigation of the deviation of the twin lamellae from $\{111\}$ at high strains. It is therefore not known for sure whether slip is restricted to the slip plane parallel to the twin lamellae as in the grains with bundles at moderate strains. However, there are very good reasons to assume that this is the case: the structural conditions with a high density of closely spaced twin lamellae are favourable for glide on one slip plane, and nobody has positively observed anything like the very large deviations from $\{111\}$ that one would get by extrapolating the calculated upper-bound deviation distribution in fig. 5 to high strains.

\section{TEXTURE-MICROSTRUCTURE RELATION IN MATERIALS WITH LOW STACKING FAULT ENERGY}

At moderate strains the restriction of slip to one slip plane in the grains with bundles is a well documented implication of the microstructural observations, and it is obviously the reason why the texture is not of the Taylor type. The situation is basically that considered in the modified Sachs model. ${ }^{16}$ Strain continuity is not maintained by a pattern where each grain follows the macroscopic strain; the heterogeneous strain (in the bundles) in one grain is accommodated by multiple slip in the neighbouring grain in a complex scheme of cooperation between the grains. Therefore, the modified Sachs model provides a rather good simulation of the experimental texture. It is evident, though, that from a theoretical point of view the present version of the modified Sachs model is only a crude approximation. A more realistic model would specifically include the two types of grains: those with bundles restricting slip to one single slip plane, and those without bundles and hence presumably without slip restrictions.

One can, at moderate strains, identify three specific mechanisms which together maintain strain continuity (all within the basic framework of the modified Sachs model):

(i) accommodation by multiple slip in the grains without bundles

(ii) heterogeneous slip on one slip plane in the grains with bundles, as reflected in the observation of bent bundles

(iii) slip on a single slip plane (in grains with bundles) which appears to be unsystematic when the grain is considered in isolation.

The exact reason why slip is restricted to one slip plane in grains with bundles is not known. It is easily understood that slip in the bundles, with their composite structure, is restricted to one slip plane, but that does not explain why slip is restricted to the bundles. There must be some condition of microscopic plastic instability which links slip to bundle formation on a specific $\{111\}$ plane. As shown by Leffers and Van Houtte, ${ }^{26}$ the orientation distribution of the twins reflects a modified-Sachs situation with one specific preferred $\{111\}$ plane already at the strain where twins/bundles start to form (rather than a Taylor situation with multiple choice of $\{111\}$ planes). We therefore suggest that modified-Sachs conditions are established at the very early stage of deformation - because of planar slip or low cross-slip frequency. This condition is then accentuated and made visible by the formation of twins/bundles.

The texture component with $\{111\}$ parallel to the rolling plane is a fascinating part of the brass-type texture. It appears as a significant texture component at reductions of $\sim 60 \%$, but at the microscopic level it is observed at substantially lower strains. ${ }^{19}$ It 
is always associated with twin lamellae parallel to the rolling plane, and there is no doubt that it is an effect of overshooting or latent hardening in the twin-matrix composite as first suggested by Kamijo and Sekine. ${ }^{27}$ From a macroscopic point of view overshooting can make $\{111\}$ approach an orientation parallel to the rolling plane, but it cannot take it the whole way to this orientation. When the orientation is actually reached, it must be a consequence of the cooperation between the grains: the local stresses deviate so much from the macroscopic stress that the orientation with $\{111\}$ parallel to the rolling plane may be reached with non-zero resolved shear stress (i.e. it is an example of mechanism (iii)). Duggan et al. ${ }^{20}$ have suggested that the difficulty of continued slip in the grains with $\{111\}$ and twin lamellae parallel to the rolling plane produce a local situation of plastic instability which is a condition for the formation of shear bands. We see an alternative possibility ${ }^{19}$ of a more macroscopic condition of plastic instability: if slip is restricted to one slip plane in all the grains by twin lamellae, this would make continued slip with preservation of strain continuity impossible (mechanism (i) is not available any more).

The processes of shear banding and subsequent slip lead to the final brass-type texture with clear maxima at the $\{110\}<112>$ positions in the pole figures. The reason why the last stage of the texture development leads to a strengthened modified-Sachs texture is not known. The way to the answer probably goes via further microstructural investigations. It has been suggested ${ }^{20,24}$ that the late stage represents the formation of the brass-type texture. This point of view is not compatible with the observation that materials with low stacking fault energy have their own development in texture (and microstructure) from the very early stage. The development of the brass-type texture really starts at strain zero.

It is interesting to think of the great number of different ideas which have, at various stages, gone into the development of the present understanding of the brass-type texture. Of the more important one can mention:

the brass-type texture is of a Sachs type - as far as the main orientations are concerned $^{28} /$ as far as the overall texture is concerned ${ }^{29}$

the brass-type texture is related to overshooting or latent hardening - within a Taylor framework, ${ }^{30} /$ by enforcing a non-Taylor deformation pattern ${ }_{20,25}$ by leading to the texture component with $\{111\}$ parallel to the rolling plane ${ }^{20,25}$

twinning is important via a volume effect ${ }^{18}$ / not via a volume effect ${ }^{23}$ / via the formation of the texture component with $\{111\}$ parallel to the rolling plane ${ }^{20}$

shear bands are important at the late stage $e^{20}$

the initial texture development is like that of the copper-type texture ${ }^{18} /$ is not like that of the copper-type texture. ${ }^{10}$

\section{TEXTURE AND MICROSTRUCTURE IN BENT BRASS PLATE}

The ultimate proof of the intimate relation between texture and microstructure 
in rolled fcc material with low stacking fault energy (if an ultimate proof is necessary) may be found in observations on a brass plate ( $15 \%$ zinc) which was unintentionally bent during rolling. 31

The brass plate was rolled to $16 \%$ reduction without bending. Then it was rolled to $38 \%$ reduction with bending (because the rolling mill could not give sufficient reduction in each pass), and therefore it had to be straightened three times. The total accumulated added strain from bending and straightening was $\sim 0.05$ in the surface to be compared with a total rolling strain of $\sim 0.5$.

From an isolated texture point of view this small added strain should not have any significant effect - particularly not when one realizes that the bending and the straightening strains are in opposite directions. In practice there was a very clear effect on texture as shown in fig. 6. The figure shows the ODF for the surface layer of the bent and straightened plate (derived from measurements with neutron diffraction) together with the ODF for the surface layer of a plate of the same material rolled to the same reduction without bending and straightening. The total thickness of the rolled plates is $7.85 \mathrm{~mm}$, and the surface specimens were $3 \mathrm{~mm}$ thick, i.e. we are not dealing with surface textures in the ordinary sense. For the plate rolled without bending there was only a small difference between the texture in the surface layer and that in the central layer. The texture in the central layer of the bent and straightened plate (with a much smaller added strain) was similar to that in the plate rolled without bending.

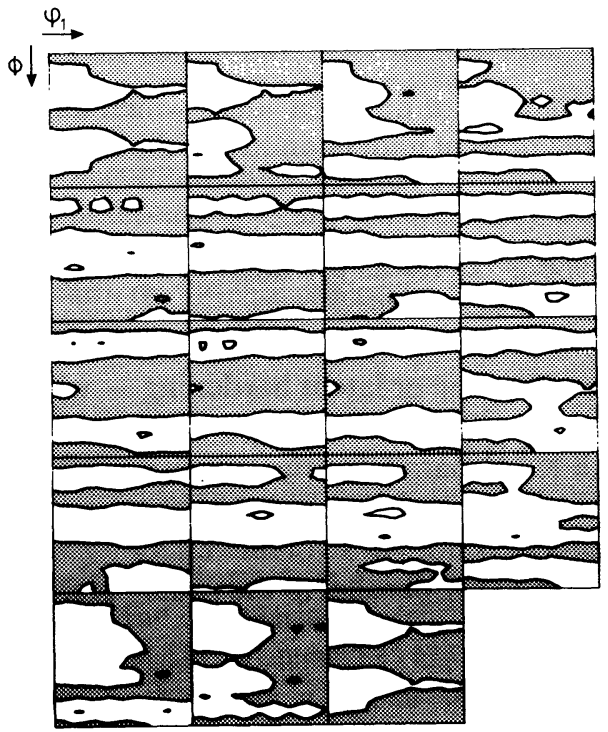

(a)

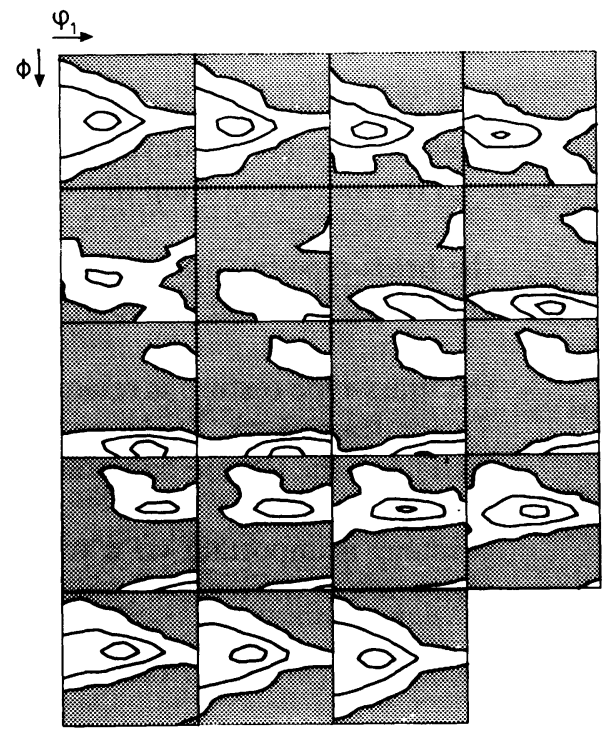

(b)

Fig. 6. ODFs for surface layers in the bent and straightened plate (a) and in normal rolled plate (b) - shown as constant $-\phi_{2}$ sections with $5^{\circ}$ interval. The contour lines correspond to orientation densities of 1,2 and 3. Areas with orientation density below unity are indicated. 


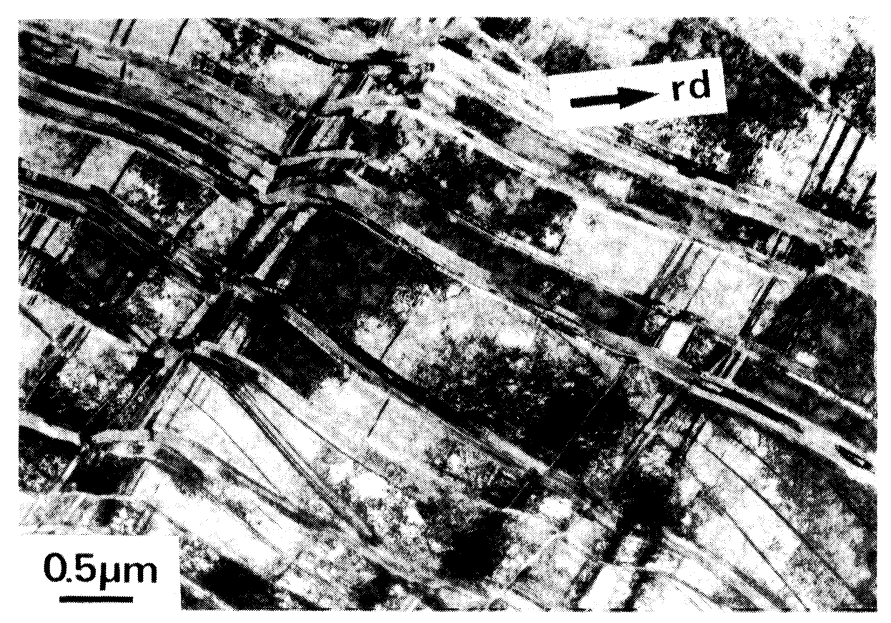

Fig. 7. Complex structure in the surface layer of the bent and straightened plate. Longitudinal section.

Like ordinary rolled brass the surface material in the bent and straightened plate consists of a mixture of grains with twins/bundles and grains without. But the grains with twins have a microstructure which is clearly different from that of the twinned grains in ordinary brass (which is fairly regular, normally with only one system of bundles as shown in fig. 4). The twinned grains in the surface layer of the bent and straightened plate have a very heterogeneous microstructure, normally with two or three systems of crossing twins/bundles in a given area as shown in fig. 7. Those in the central layer have a normal microstructure.

It is obvious what happens. There is a normal brass development in texture and microstructure up to $16 \%$ reduction (where bending starts). For some reason bending and straightening disturb the continued development of the simple bundle structure with slip on one single slip plane which is the condition for the normal texture development in brass. This changes the course of the texture development - towards some other type of texture. The very low orientation densities in fig. $6 \mathrm{a}$ reflect the change in course: the texture in fig. 6 a results from the superposition of two different texture developments.

Thus, by changing the microstructure a small additional strain, which would otherwise be insignificant, can produce dramatic changes in texture.

\section{TEXTURES IN MATERIALS WITH HIGHER STACKING FAULT ENERGIES}

It is normally stated that the rolling texture in fcc materials with high and intermediate stacking fault energies follows the Taylor model with appropriately relaxed constraints in accordance with the grain shape, e.g. refs. ${ }^{2,32}$. However, this statement should be taken with some qualification. For instance there is a clear trend that the experimental textures at moderate strains are not full-constraint textures - as they should be from a theoretical point of view since the grains do not deviate very much from an equiaxed shape. 
Fig. 8 shows $\{111\}$ and $\{200\}$ pole figures for $50 \%$ reduction simulated with the full-constraint Taylor model together with experimental copper pole figures. In the simulated $\{111\}$ pole figure the intensity maximum close to $R D$ is removed from the periphery, whereas it is at the periphery in the experimental pole figure - as it is in relaxed-constraint simulated pole figures. The same difference was reported for $37 \%$ reduction in ref. ${ }^{3}$. The simulated pole figures in fig. 8 are produced by one of the present authors, but pole figures simulated by $B$. Bacroix and by $T$. Lowe are practically identical to those in fig. $8 .{ }^{33}$ The experimental pole figures in fig. 8 are similar to other experimental pole figures for copper rolled to moderate reductions, e.g. refs. ${ }^{34,35}$ It should be mentioned that there is, in the literature (including these proceedings), a number of independent reports of relaxed-constraint behaviour without justification in the grain shape - which we shall not quote here.

$\mathrm{RD}$

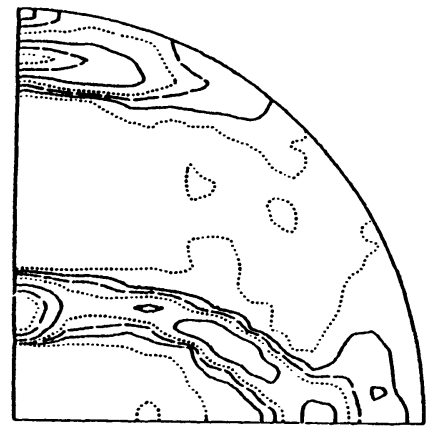

111

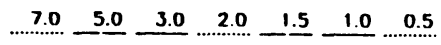

$\mathrm{RD}$

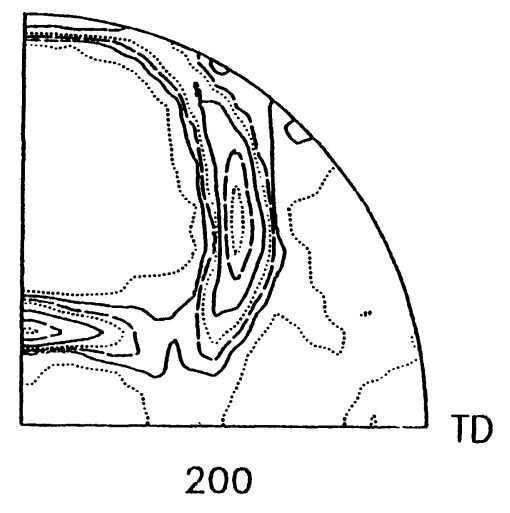

$7.0 .0 \quad 5.0 \quad 3.0 \quad 2.0 \quad 1.5 \quad 1.0 \quad 0.5$
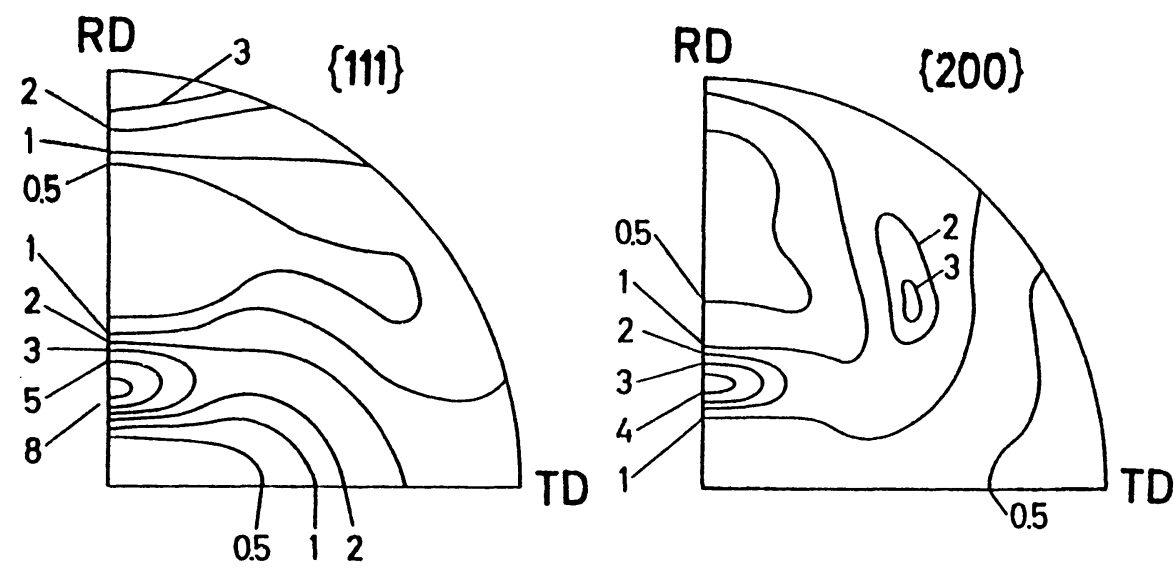

Fig. 8. $\{111\}$ and $\{200\}$ pole figures for $50 \%$ rolling reduction. Top: simulated with the full-constraint Taylor model; bottom: measured for copper. 
The pole figures in fig. 8 show another significant and well known difference between the simulated and the experimental texture: the pole densities generally reach much higher values in the simulated than in the experimental pole figures (as opposed to fig. 3 where there is a reasonable quantitative agreement).

There are other deviations from the texture pattern derived from the simple full-constraint/relaxed-constraint Taylor model than those seen in fig. 8. At very high strains one would expect a clean relaxed-constraint texture as observed (approximately) for copper, whereas the high-strain aluminium rolling texture is somewhere between a relaxed-constraint and a full-constraint texture. ${ }^{34}$ The relatively strong $\{110\}<112>$ component in the experimental copper-type textures is not predicted in the model. ${ }^{5}$ The Taylor model does not provide for any grain-size effect on texture, but actually there are various effects of grain size; for instance the development of rolling texture in copper $^{35}$ and aluminium ${ }^{36}$ is slower in coarse-grained than in fine-grained materials.

As our conclusion we may state that even though the Taylor model complex provides a reasonable description of rolling textures in fcc materials with high and intermediate stacking fault energies, there are a number of details which do not really fit into the existing framework. In the next section we shall describe microstructural evolutions of a complexity far beyond that considered in the Taylor model. It is therefore not too surprising that the Taylor model falls short of describing all the details in the textural evolution correctly.

\section{MICROSTRUCTURES IN MATERIALS WITH HIGHER STACKING FAULT ENERGIES}

Aluminium and copper are the fcc materials with high to intermediate stacking fault energy for which the microstructural evolution during rolling deformation has been most extensively studied, and they are the materials we shall deal with. The microstructures observed in nickel are not fundamentally different from those observed in aluminium and copper. ${ }^{39}$

The concept of microstructure covers all aspects of the dislocation arrangements. The arrangement of the dislocations in cells or subgrains is the fine-scale end of the range of microstructures. For most practical purposes the cells/subgrains are, with our present knowledge, below the resolution limit of existing texture models as already mentioned in the introduction. Therefore, we shall focus on the microstructural features at coarser scale.

At the coarse-scale end of the range of microstructures we find the grain-scale microstructural variations. By this we mean variations with a wave length of the order of the grain size. When we use the wave analogy, we are not necessarily talking about any regular wave shape; for instance we do not exclude that the variations concentrate near the grain boundaries. Basically, we are dealing with variations in the dislocation structure. A map of the distribution of the dislocations including their Burgers vectors would contain the complete information. But we do not have such maps, and therefore we have to use indirect indicators such as variation in lattice orientation, variation in cell size and shape, variations in strain as measured on the surface, variations in the pattern of surface slip markings. The variations may be smooth, or they may be localized 
to produce abrupt changes. The latter are of course most easily recognizable in the microstructure. Boas and Hargreaves ${ }^{40}$ provided the classical example of grain-scale variations - in strain as measured on the surface of tensile-deformed coarse-grained aluminium.

Bay and Hansen ${ }^{41}$ and Barlow, Bay and Hansen ${ }^{42}$ have investigated the grainscale variations in coarse-grained and fine-grained aluminium rolled to reductions up to $30 \%$. The main observations may be summarized as follows: there are banded structures along many grain boundaries; there are cumulative changes in lattice orientation across the banded structures (over distances of $10 \mu \mathrm{m}$ or more from the boundaries); close to some grain boundaries the cell size is different from that in the grain interior; the pattern of surface slip bands in the vicinity of many grain boundaries deviates from that in the grain interior. One of the present authors ${ }^{17}$ found large grain-scale microstructural variations in copper rolled to reductions up to $50 \%$ : cumulative changes in lattice orientation throughout the average grain but most pronounced near the grain boundaries, occasionally subdivision in deformation bands, banded structures along many grain boundaries, deviation in the slip-line pattern on the surface in the vicinity of many grain boundaries.

The most intriguing of the microstructural features are the "organized structures $^{n 3}$ at a scale intermediate between that of the grain-scale variations and that of cells/subgrains. They may actually have grain dimensions in two directions, but they are always much smaller than the grain size in one direction, and they repeat themselves in this direction. The first example to be observed was the banded structure in copper. ${ }^{43,44}$

For the organized structures in aluminium we shall quote the recent investigatioan by Bay, Hansen and Kuhlmann-Wilsdorf. ${ }^{45}$ The first microstructural feature to appear as part of the organized structure is a system of parallel dense dislocation walls (DDWs). They are sharp wall segments of dimensions of several to many cell diameters with an associated orientation change which is much larger than that associated with the cell walls. At about $10 \%$ reduction sections of the DDWs transform to become first-generation microbands (MBs). The MBs are flat three-dimensional entities consisting of small pancake-shaped cells (SPCs). The fraction of DDWs transformed to MBs increases with strain. The DDWs/MBs subdivide the grains in cell blocks (CBs) with relatively large orientation differences (typically $\sim 10^{\circ}$ at $30 \%$ reduction) and hence with different combinations of active slip systems. Normally there is a $+/$ - relation between the orientation change across neighbouring sets of DDWs/MBs which, as a first approximation, means that a system of parallel DDWs/MBs define two families of CBs. The orientation of the DDWs/MBs is approximately parallel to the transverse direction making an angle of $\sim 35^{\circ}$ with the rolling direction. This orientation seems to be determined by macroscopic geometry and not by crystallography. Initially there is only one set of parallel DDWs/MBs, but at reductions of $\sim 30 \%$ a second, symmetrical set forms in many grains. This situation is sketched in fig. 9. At high reductions $(\sim 85 \%)$ a new set of DDWs/MBs, with orientations approximately parallel to the rolling direction, is observed in many grains. ${ }^{46}$ Ref. ${ }^{46}$ included materials with different grain sizes (range 35-400 $\mu \mathrm{m}$ ), and it was observed that the dimensions of the DDWs/MBs do not scale with grain size; the variation by a factor of $\sim 10$ in grain size resulted in a variation in the dimensions of the DDWs/MBs by a factor of $\sim 2$. It was also observed that the DDW/MB structure was less developed in the fine-grained material. 


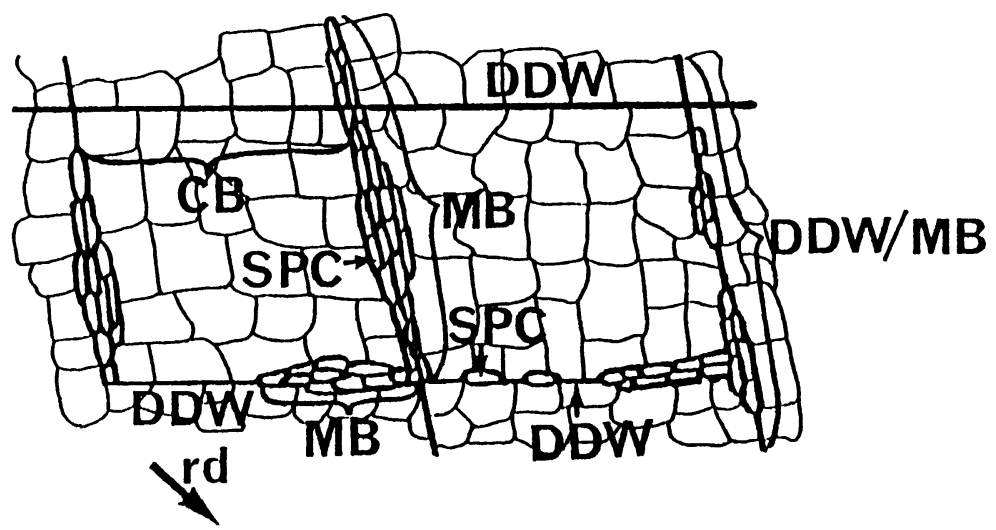

Fig. 9. Sketch of the microstucture of aluminium as described in ref. ${ }^{45}$ (seen in longitudinal section). The actual drawing is from ref. ${ }^{3}$

The second-generation microbands in copper ${ }^{43,44,47,48}$ have a microstructure and a micromechanical function different from those of the first-generation microbands in aluminium. They consist of two sets of dislocation walls approximately parallel to $\{111\}$ enclosing an area with a rather low dislocation density. In agreement with various earlier suggestions Ananthan et $\mathrm{al}^{47,48}$ demonstrated that the second-generation microbands tended to "evaporate", i.e. they have a low structural stability. The primary function of the second-generation microbands is not to separate areas with different combinations of slip systems. The shear offsets produced when they cross other microstructural elements show that they are carriers of concentrated shear - as the bundles in materials with low stacking fault energy. After the first observations of (secondgeneration) microbands in copper it has normally been assumed (e.g. refs. ${ }^{43,44,49,50}$ ) that all plate-shaped structures in copper are of this type. More recent investigations $^{47,48}$ have shown that rolled copper also contains dense dislocation walls and first-generation microbands: a substantial fraction of the grains are subdivided in small areas with uniform lattice orientation by two sets of dislocation walls as shown in fig. 10 . At places the dislocation walls split into two parallel walls, thereby forming first-generation mirobands. The dense dislocation walls/first-generation microbands in copper have macroscopically determined (non-crystallographic) orientations similar to those of their counterparts in aluminium. The grains which do not develop the type of microstructure shown in fig. 10 have an equiaxed cell structure. The distribution of second-generation microbands is described in detail in refs. ${ }^{46,47}$ So far, there are no established simple crystallographic rules which can account for the formation of second-generation microbands. ${ }^{21,50}$ The wall and cell structures are well developed at a reduction of $10 \%$. Second-generation microbands are observed in small numbers already at $5 \%$ reduction and in large numbers at reductions $\geq 20 \%$, frequently in two different systems. At high strains (reductions $>60 \%$ ) banded features approximately parallel to the rolling plane dominate the microstructure as already observed by Malin and Hatherly. ${ }^{43}$ Ananthan et al. ${ }^{48}$ suggest that they are dislocation walls formed as such and not rotated second- generation microbands as suggested earlier. ${ }^{43,44}$ 


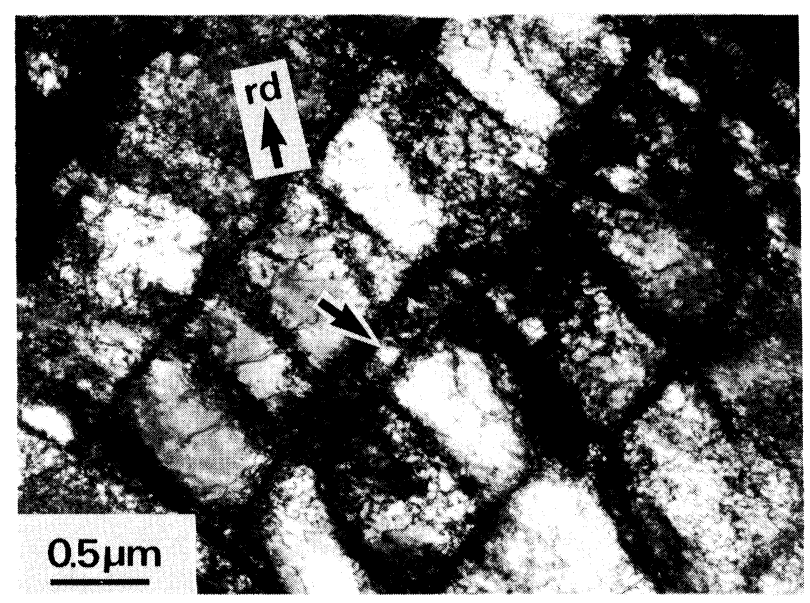

Fig. 10. Dense dislocation walls and firstgeneration microband (marked with arrow) in copper rolled to $20 \%$ reduction, longitudinal section. By courtesy of V.S. Ananthan.

\section{TEXTURE-MICROSTRUCTURE RELATION IN MATERIALS WITH HIGHER STACKING FAULT ENERGY}

The origin of the grain-scale variations in microstructure is clear; as described many times before, e.g. refs., $3,16,51$ they reflect the intergranular accommodation processes. All deformation models with softer grain-to-grain (or grain-to-matrix) interaction than that derived from the elastic constants imply grain-scale variations. The basic types of models are relaxed-constraint models (relaxed according to grain shape $e^{2,32}$ or relaxed for other reasons ${ }^{17}$ ) and self-consistent models, e.g. refs., $51,52,53$ but there are a number of variations, e.g. refs. ${ }^{54,55,56}$ Obviously one should not, as it was the case for materials with low stacking fault energy, go to extreme soft-interaction models like the modified Sachs model. Most of these models deal with homogeneously deforming individual grains interacting with a continuum matrix, while some deal with homogeneously deforming individual grains interacting with each other according to some statistical scheme. Translation to the real world of plastically interacting grains implies intragranular variations in strain and slip pattern and hence in microstructure. Finiteelement models include the intragranular variations in a direct way, e.g. ref. ${ }^{57} \mathrm{We}$ shall not go into details about the different models but just quote that there is, within the range of models, the capacity to produce simulated textures which agree better with the experimental textures than those produced by the Taylor model - i.e. to help removing the inconsistencies described in the section about the textures. Qualitatively there is thus a perfect agreement between texture and the grain-scale variations in microstructure: both point at deformation models with soft interaction.

The grain-scale variations in microstructure are imposed directly by the polycrystalline nature of the materials, and their function is to maintain continuity. We suggest a different (but not necessarily independent) function for the organized structures: they take part in the process of executing the local strain in the most efficient way under the given imposed conditions. For instance, Bay et $\mathrm{al}^{45}$ have suggested that subdivision in 
cell blocks reduces work hardening (by reducing the number of active slip systems at any given spot). Thus, organized structures may exist independently of grain-scale variations in microstructure (as they actually do in deformed single crystals, e.g. ref. ${ }^{58}$ ). Within this framework one can imagine a strong effect of the organized structures on texture since they are connected to the microscopic slip pattern which produces the texture. In materials with low stacking fault energy we have seen an example of such a strong effect. The problem is that so little is known about the detailed mechanisms leading to the formation of the organised structures in materials with higher stacking fault energy, and therefore we have to make do with hand-waving suggestions.

For the subdivision in cell blocks with different slip patterns one must assume that it scatters the texture since the different cell blocks will take up different orientations. It has been argued ${ }^{3}$ that plate-shaped cell blocks may impose relaxed constraints at the early stage of deformation where the grain- shape argument for relaxed constraint does not work. Thus, the cell blocks may be part of the explanation for the differences between calculated and experimental textures as discussed in connection with fig. 8 (the experimental texture is less sharp than the calculated texture and more in the direction of relaxed constraints). The observed grain-size effect on the band/wall structure in aluminium $^{46}$ shows that the organized structure does not scale with grain size. Microstructural and micromechanical parameters which do not scale with grain size are exactly what we need in order to explain the grain-size effect on texture. ${ }^{59}$ Qualitatively the argument works out correctly: the organized structure is more developed in coarsegrained materials and the organized structure scatters the texture, hence the delayed texture development in coarse-grained materials.

For the second-generation microbands their orientation quite close to $\{111\}^{48}$ might suggest that they reflect a strong preference for slip on one single slip plane, cf. the discussion in connection with fig. 5. But, as argued by Ananthan et al. ${ }^{48}$ this is probably not the case; the second-generation microbands simply disappear before they rotate significantly away from their parent $\{111\}$ plane. Altogether, the structural instability of the second-generation microbands makes it difficult to assess the role they play; for instance the magnitude of their contribution to the total shear is uncertain.

There is no reason to doubt that the organized structures, particularly the subdivision in cell blocks, represent clear local deviations from the slip patterns considered in the existing models. When the effects on texture are not very obvious, the reason must be that texture formation under polyslip conditions is not very sensitive to the details of the slip pattern. The difference in organized structure between aluminium and copper would be an obvious explanation for the difference in texture.

Quantitative investigations of the microstructures (both grain-scale variation and organized structure) are necessary to bring us to the point where we really understand the texture-microstructure relation - and thereby the deformation process.

\section{CONCLUDING REMARKS}

For materials with low stacking fault energy we have demonstrated that texture and microstructure taken together provide an appropriate empirical description of the process of plastic deformation - even though a number of "details", including the 
fundamental underlying dislocation processes, need to be sorted out. For materials with higher stacking fault energies, the picture is less clear, but it is obvious that we have to combine texture and microstructure before we can claim to know what is really going on during plastic deformation.

Thus, a proper understanding of materials behaviour requires that we consider texture and microstructure as two sides of the same problem. And we do not refer to understanding in an abstract academic sense, but to the understanding necessary to make the most efficient use of our materials. For instance, it must by now be considered an established fact, e.g. refs., ${ }^{460}$ that mechanical anisotropy is produced by a combination of texture and microstructure (and not by texture alone as previously assumed). Recrystallization is another area of great technological importance which depends on a combination of texture and microstructure. An obvious implication would be that we attempt to extend the scope of texture simulation to cover simultaneous simulation of texture and microstructure. ${ }^{26,33}$

As a final remark we can mention that we have adopted the traditional grouping of the fcc materials according to their stacking fault energy. There are a number of indications, e.g. ref., ${ }^{61}$ that short-range ordering, rather than stacking fault energy, determines the mechanical behaviour. In the present context it is not particularly important whether the effect of alloy atoms comes via a decreased stacking fault energy or via an increased trend to form short-range order - as long as the basic effect is not questioned, viz. the increase in the trend for planar slip.

\section{ACKNOWLEDGEMENTS}

Thanks are due to V.S. Ananthan, B. Bay and N. Hansen for helpful discussions and, not the least, to L. Jørgensen for typing the various versions of the manuscript. Financial support from COWIfonden is gratefully acknowledged.

\section{REFERENCES}

1. G.I. Taylor, J. Inst. Met. 62, 307 (1938).

2. H. Mecking, in "Proc. ICSMA 5" (P. Haasen et al., eds., Pergamon Press, Oxford 1980) p. 1573.

3. T. Leffers and N. Hansen, in "Materials Architecture" (J. B. Bilde-Sørensen et al., eds., Risø National Laboratory 1989) p. 127.

4. J. Hirsch and K. Lücke, Acta metall. 36, 2863 (1988).

5. J. Hirsch and K. Lücke, Acta metall. 36, 2883 (1988).

6. J. Hirsch, K. Lücke and M. Hatherly, Acta metall. 36, 2905 (1988).

7. W. Truszkowski, J. Dutkiewicz and J. Szpunar, Bull. Acad. Polon. Sci. 17 (6), 53 (1969).

8. T. Leffers and P. Kayworth, in "Proc. ICOTOM 3" (Société Francaise de Métallurgie, Paris 1973) p. 149.

9. J. Tobisch and A. Mücklich, Texture, 1, 211 (1974).

10. T. Leffers and D. Juul Jensen, Text. Microstruct. 8/9, 467 (1988).

11. J. Gryziecki, W. Truszkowski, J. Pospiech and J. Jura, in these proceedings.

12. C. Donadille, R. Valle, P. Dervin and R. Penelle, Acta metall. 37, 1547 (1989).

13. J.S. Kallend and G.J. Davies, Phil. Mag. 25, 471 (1972).

14. P. Van Houtte, Acta metall. 26, 591 (1978). 
15. G. Sachs, Z. verein. deut. Ing. 72, 734 (1928).

16. O.B.Pedersen and T. Leffers, in "Constitutive Relations" (S.I. Andersen et al., eds., Risø National Laboratory 1987) p. 147.

17. T. Leffers, Risø Report No. 302 (1975).

18. G. Wassermann, Z. Metallkde. 54, 61 (1963).

19. T. Leffers and J.B. Bilde-Sørensen, Acta metall. mater. 38, 1917 (1990).

20. B.J. Duggan, M. Hatherly, W.B. Hutchinson and P.T. Wakefield, Metals Sci. 12, 343 (1978).

21. T. Leffers and V.S. Ananthan, in these proceedings.

22. M. Blicharski and S. Gorczyca, Metals Sci. 12, 303 (1978).

23. T. Leffers and A. Grum Jensen, Trans. Met. Soc. AIME 242, 314 (1968).

24. W.B. Hutchinson, B.J. Duggan and M. Hatherly, Metals Technol. 6, 398 (1979).

25. B. Fargette and D. Whitwham, Mém. Sci. Rev. Métall. 73, 197 (1976).

26. T. Leffers and P. Van Houtte, Acta metall. 37, 1191 (1989).

27. T. Kamijo and K. Sekine, Met. Trans. 1, 1287 (1970).

28. I.L. Dillamore and W.T. Roberts, Acta metall. 12, 281 (1964).

29. T. Leffers, Phys. Stat. Sol. 25, 337 (1968).

30. J.F.W. Bishop, J. Mech. Phys. Sol. 3, 130 (1954).

31. T. Leffers and D. Juul Jensen, Scripta metall. mater. (in press).

32. U.F. Kocks and G.R. Canova, in "Deformations of Polycrystals" (N. Hansen et al., eds., Risø National Laboratory 1981) p. 35.

33. T. Leffers, in "Proc. ICOTOM 8" (J.S. Kallend and G. Gottstein, eds., The Metallurgical Society, Warrendale 1988) p. 273.

34. W. Truszkowski and J. Krol, Mém. Sci. Rev. Mét. 65, 907 (1968).

35. J.S. Kallend and G.J. Davies, Texture, 1, 51 (1972).

36. P. Van Houtte, in "Proc. ICOTOM 6" (S. Nagashima, ed., The Iron and Steel Institute of Japan, Tokyo 1981) p. 428.

37. T. Öztürk, J.S. Kallend and G.J. Davies, in "Proc. ICOTOM 6" (S. Nagashima, ed., The Iron and Steel Institute of Japan, Tokyo 1981) p. 507.

38. N. Hansen, B, Bay, D. Juul Jensen and T. Leffers, in "Proc. ICSMA 7" (H.J. McQueen et al., eds., Pergamon Press, Oxford 1985) p. 317.

39. B. Bay, N. Hansen, D.A. Hughes and D. Kuhlmann-Wilsdorf, submitted for publication.

40. W. Boas and M.F. Hargreaves, Proc. Roy. Soc. 193A, 89 (1948).

41. B. Bay and N. Hansen, in "Deformation of Polycrystals" (N. Hansen et al., eds., Risø National Laboratory 1981) p. 137.

42. C.Y. Barlow, B. Bay and N. Hansen, Phil. Mag. 51, 253 (1985).

43. A.S. Malin and M. Hatherly, Metals Sci. 13, 463 (1979).

44. M.Hatherly, in "Proc. ICSMA6" (R.C. Gifkins, ed., Pergamon Press, Oxford 1983) p. 1181.

45. B. Bay, N. Hansen and D. Kuhlmann-Wilsdorf, Mater. Sci. Eng. A113, 385 (1989).

46. D. Juul Jensen and N. Hansen, Acta metall. mater. 38, 1369 (1990).

47. V.S. Ananthan, T. Leffers and N. Hansen, Mater. Sci. Techn. (in press).

48. V.S. Ananthan, T. Leffers and N. Hansen, Scripta metall. mater. (in press).

49. F.J. Torrealdea and J. Gil Sevillano, in "Proc. ICSMA 6" (R.C. Gifkins, ed., Pergamon Press, Oxford 1982) p. 547.

50. E. Nes, W.B. Hutchinson and A.A. Ridha, in "Proc. ICSMA 7" (H.J. McQueen et al., eds., Pergamon Press, Oxford 1985) p. 57.

51. S. Tiem, M. Berveiller and G.R. Canova, Acta metall. 34, 2139 (1986). 
52. M. Berveiller, A. Hihi and A. Zaoui, in "Deformation of Polycrystals" (N. Hansen et al., eds., Risø National Laboratory 1981) p. 145.

53. A. Molinari, G.R. Canova and S. Ahzi, Acta metall. 35, 2983 (1987).

54. M. Arminjon, Acta metall. 35, 615 (1987).

55. R. Fortunier and J.H. Driver, Acta metall. 35, 1355 (1987).

56. B.L. Adams, G.R. Canova and A. Molinari, Text. Microstruct. 11, 57 (1989).

57. S.V. Harren and R.J. Asaro, J. Mech. Phys. Sol. 37, 191 (1989).

58. Y. Kawasaki and T. Takeuchi, Scripta metall. 14, 342 (1980).

59. T. Leffers, D. Juul Jensen and N. Hansen, in "Proc. ICOTOM 8" (J.S. Kallend and G. Gottstein, eds., The Metallurgical Society, Warrendale 1988) p. 449.

60. J.L. Raphanel, J.-H. Schmitt and B. Baudelet, in "Constitutive Relations" (S.I. Andersen et al., eds., Risø National Laboratory 1987) p. 491.

61. V. Gerold and H.P. Karnthaler, Acta metall. 37, 2177 (1989). 